\title{
Stabilization of complex-valued neural networks with time-varying delays via linear feedback control
}

\author{
Zhenjiang Zhao, a and Qiankun Song ${ }^{2, b}$ \\ ${ }^{1}$ Department of Mathematics, Huzhou University, Huzhou 313000, China \\ 2 Department of Mathematics, Chongqing Jiaotong University, Chongqing 400074, China \\ azhaozjcn@163.com, bqiankunsong@163.com
}

\begin{abstract}
Keywords: Complex-valued neural networks, delay, stability, complex-valued linear matrix inequality.
\end{abstract}

\begin{abstract}
In this paper, a class of complex-valued neural networks with time-varying delays is considered without assuming the differentiability of the time-varying delays, and the exponential stabilization for the considered neural networks is investigated. By constructing proper Lyapunov-Krasovskii functional and using the matrix inequality techniques, a delay-dependent criterion for checking the stability of the considered neural networks is presented under designed linear feedback controller. An example with simulations is given to show the effectiveness of the obtained result.
\end{abstract}

\section{Introduction}

In the past decade, delayed neural networks have been successfully applied in many areas such as signal processing, pattern recognition, associative memories, and optimization solvers [1]. Some of these applications require the designed neural network to be stable, and it is therefore important to study the stability of neural networks [2]. A great number of results have been reported on the stability for various neural networks with constant delays or time-varying delays in the literature for example, see $[1,2,3,4,5,6]$ and references therein.

As an extension of real-valued neural networks, complex-valued neural networks with complex-valued state, output, connection weight, and activation function become strongly desired because of their practical applications in physical systems dealing with electromagnetic, light, ultrasonic, and quantum waves[7]. In fact, complex-valued neural networks (CVNNs) make it possible to solve some problems which cannot be solved with their real-valued counterparts. For example, the XOR problem and the detection of symmetry problem cannot be solved with a single real-valued neuron, but they can be solved with a single complex-valued neuron with the orthogonal decision boundaries, which reveals the potent computational power of complex-valued neurons[8]. Besides, CVNNs has more different and more complicated properties than the real-valued ones [9]. Therefore it is necessary to study the dynamic behaviors of CVNNs deeply [10]. Recently, some stability results of CVNNs have been obtained, for example, see [7, 8, 9, 10, 11, 12, 13, 14, 15, 16, 17, $18,19,20,21,22]$ and references therein.

In practice, it may happen that the delayed neural networks are unstable or the convergence rate can not meet the requirements. Under this case, certain controllers may be designed such that the controlled delayed neural networks achieve the desired stability properties. In recent years, many control approaches have been developed to stabilize chaotic complex-valued neural networks such as adaptive control, fuzzy control, sampled-data control, impulsive control and intermittent control and so on. However, To the best of our knowledge, up to now, there are very few results on the stabilization problem of CVNNs.

Motivated by the above discussions, the objective of this paper is to study the exponential stabilization of CVNNs with time-varying delays. 


\section{Preliminary}

In this paper, we consider the following CVNNs with time-varying delays

$$
\dot{x}(t)=-D x(t)+A f(x(t))+B f(x(t-\tau(t)))+u(t)
$$

for $t \geq t_{0}$, where $x(t)$ is the state vector of the network at time $t, n$ corresponds to the number of neurons; $D$ is a positive diagonal matrix, $A$ and $\mathrm{B}$ are known constant matrices; $f(x(t))$ denotes the neuron activation at time $t ; u(t)$ is a external input vector; $\tau(t)$ is delays and satisfy $0 \leq \tau(t) \leq \tau$. To order to stabilize the origin of neural networks (1) by means of feedback control, we assume that the control exposed on the system is of the form

$$
u(t)=K x(t)
$$

where $K$ is the control gain matrix. With control law (2), model (1) can be rewritten as

$$
\dot{x}(t)=(K-D) x(t)+A f(x(t))+B f(x(t-\tau(t)))
$$

Model (1) is supplemented with initial value given by $x(s)=\varphi(s), s \in\left[-\tau, t_{0}\right]$, where is bounded and continuously differential on $s \in\left[-\tau, t_{0}\right]$.

Throughout this paper, we make the following assumption:

Assumption 1. For any $j=1,2, \cdots, n, f_{j}(0)=0$ and there exists a positive diagonal matrix $L=\operatorname{diag}\left(l_{1}, l_{2}, \cdots, l_{n}\right)$ such that for any $x, y \in C$,

$$
\left|f_{j}(x)-f_{j}(y)\right| \leq l_{j}|x-y|
$$

\section{Main Results}

Theorem 1. Assume that the assumption 1 holds. For viven constant $\alpha>0$, if there exist three symmetric positive definite Hermitian matrices $P_{1}, P_{2}$ and $P_{3}$, two positive diagonal matrices $R$ and $S$, and four matrices $Q_{1}, Q_{2}, W$ and $Z$ such that the following complex-valued linear matrix inequality holds:

$$
\Omega=\left(\Omega_{i j}\right)_{8 \times 8}<0
$$

Where

$\Omega_{i j}=\Omega_{j i}$, and

$\Omega_{11}=\alpha P_{1}+P_{2}-Q_{1}-Q_{1}^{*}+L R L+Z-W D+Z^{*}-D W^{*}, \Omega_{12}=P_{1}+Z^{*}-D W^{*}-W, \Omega_{13}=Q_{1}$,

$\Omega_{15}=W A, \Omega_{16}=W B, \Omega_{17}=Q_{1}, \Omega_{22}=\tau e^{\alpha \tau} P_{2}-W-W^{*}, \Omega_{25}=W A, \Omega_{25}=W A$,

$\Omega_{28}=Q_{2}, \Omega_{33}=-Q_{2}-Q_{2}^{*}+L S L, \Omega_{34}=Q_{2}, \Omega_{38}=Q_{2}, \Omega_{44}=-\tau e^{-\alpha \tau} P_{2}, \Omega_{55}=-R, \Omega_{66}=-S$,

$\Omega_{77}=-P_{3}, \Omega_{88}=-P_{3}$, and the rest of $\Omega_{i j}$ are zero, then the origin of system (3) is globally exponerntially stable, and the gain matrix of control law (2) is

$$
K=W^{-1} Z
$$

Proof. Consider the following Lyapunov-Krasovskii functional as

$$
V(t)=V_{1}(t)+V_{2}(t)+V_{3}(t)
$$

where

$$
\begin{aligned}
& V_{1}(t)=x^{*}(t) P_{1} x(t) \\
& V_{2}(t)=\int_{t-\tau}^{t} e^{\alpha(s-t)} x^{*}(s) P_{2} x(s) d s \\
& V_{3}(t)=\int_{-\tau}^{0} d \xi \int_{t+\xi}^{t} e^{\alpha(s-t)} \dot{x}^{*}(s) P_{3} \dot{x}(s) d s
\end{aligned}
$$

Calculating the time derivative of $V(t)$, we obtain

$$
\begin{aligned}
\dot{V}_{1}(t) & =x^{*}(t) P \dot{x}(t)+\dot{x}^{*}(t) P_{1} x(t) \\
& =-\alpha V_{1}(t)+\alpha x^{*}(t) P_{1} x(t)+x^{*}(t) P_{1} \dot{x}(t)+\dot{x}^{*}(t) P_{1} x(t)
\end{aligned}
$$




$$
\begin{aligned}
\dot{V}_{2}(t) & =-\alpha V_{2}(t)+x^{*}(t) P_{2} x(t)-e^{-\alpha \tau} x^{*}(t-\tau) P_{2} x(t-\tau) \\
\dot{V}_{3}(t) & =-\alpha V_{3}(t)+\tau e^{\alpha \tau} \dot{x}^{*}(t) P_{3} \dot{x}(t)-\int_{-\tau}^{0} e^{\alpha \xi} x^{*}(t+\xi) P_{3} x(t+\xi) d \xi \\
& \leq-\alpha V_{3}(t)+\tau e^{\alpha \tau} \dot{x}^{*}(t) P_{3} \dot{x}(t)-\int_{t-\tau}^{t} x^{*}(s) P_{3} x(s) d s
\end{aligned}
$$

It follows from (10)-(12) that

$$
\begin{aligned}
\dot{V}(t) \leq & -\alpha V(t)+x^{*}(t)\left(\alpha P_{1}+P_{2}\right) x(t)+x^{*}(t) P_{1} \dot{x}(t)+\dot{x}^{*}(t) P_{1} x(t) \\
& -e^{-\alpha \tau} x^{*}(t-\tau) P_{2} x(t-\tau)+\tau e^{\alpha \tau} \dot{x}^{*}(t) P_{3} \dot{x}(t)-\int_{t-\tau}^{t} x^{*}(s) P_{3} x(s) d s
\end{aligned}
$$

By Newton-Leibniz formulation, we have

$$
\begin{aligned}
0= & x^{*}(t) Q_{1}\left[-x(t)+x(t-\tau(t))+\int_{t-\tau(t)}^{t} \dot{x}(s) d s\right] \\
& +\left[-x(t)+x(t-\tau(t))+\int_{t-\tau(t)}^{t} \dot{x}(s) d s\right]^{*} Q_{1} x(t) \\
\leq & x^{*}(t)\left[-Q_{1}-Q_{1}^{*}+Q_{1} P_{3}^{-1} Q_{1}^{*}\right] x(t)+x^{*}(t) Q_{1} x(t-\tau(t)) \\
& +x^{*}(t-\tau(t)) Q_{1}^{*} x(t)+\int_{t-\tau(t)}^{t} \dot{x}^{*}(s) P_{3} \dot{x}(s) d s \\
0= & x^{*}(t-\tau(t)) Q_{2}\left[-x(t-\tau(t))+x(t-\tau)+\int_{t-\tau}^{t-\tau(t)} \dot{x}(s) d s\right] \\
& +\left[x(t-\tau)-x(t-\tau(t))-\int_{t-\tau}^{t-\tau(t)} \dot{x}(s) d s\right]^{*} Q_{2} x(t-\tau(t)) \\
\leq & x^{*}(t-\tau(t))\left[-Q_{2}-Q_{2}^{*}+Q_{2} P_{3}^{-1} Q_{2}^{*}\right] x(t-\tau(t)) \\
& +x^{*}(t-\tau(t)) Q_{2} x(t-\tau)+x^{*}(t-\tau) Q_{2}^{*} x(t-\tau(t)) \\
& +\int_{t-\tau}^{t-\tau(t)} \dot{x}^{*}(s) P_{3} \dot{x}(s) d s
\end{aligned}
$$

In addition, we can obtain from Assumption 1 that

$$
\begin{aligned}
& f^{*}(x(t)) R f(x(t)) \leq x(t) \operatorname{LRLx}(t) \\
& f^{*}(x(t-\tau)) S f(x(t-\tau)) \leq x(t-\tau) \operatorname{LSLx}(t-\tau)
\end{aligned}
$$

From model (3), we have that

$$
\begin{aligned}
& 0=\dot{x}^{*}(t) W[-\dot{x}(t)+(K-D) x(t)+A f(x(t))+B f(x(t-\tau(t)))] \\
& +[-\dot{x}(t)+(K-D) x(t)+A f(x(t))+B f(x(t-\tau(t)))]^{*} W^{*} \dot{x}(t)
\end{aligned}
$$

It follows from inequality (13)-(18) that

$$
\dot{V}(t) \leq-\alpha V(t)+\eta^{*}(t) \Theta \eta(t)
$$

where $\eta(t)=\left(x^{*}(t), \dot{x}^{*}(t), x^{*}(t-\tau(t)), x^{*}(t-\tau), f^{*}(x(t)), f^{*}(x(t-\tau))\right)^{*}, \quad$ and $\quad \Theta=\left(\Theta_{i j}\right)_{6 \times 6} \quad$ with $\Theta_{11}=\Omega_{11}+Q_{1} P_{3}^{-1} Q_{1}^{*}, \Theta_{33}=\Omega_{33}+Q_{2} P_{3}^{-1} Q_{2}^{*}$, the rest of $\Theta_{i j}$ are $\Omega_{i j}$.

From Schur theorem, we know that $\Omega<0$ is equivalent to $\Theta<0$. Therefore, we have from inequality (5) and (20) that

$$
\dot{V}(t) \leq-\alpha V(t)
$$

Thus, $V(t) \leq V\left(t_{0}\right) e^{-\alpha\left(t-t_{0}\right)}$ for any $t \geq t_{0}$. From the definition of $V(t)$, we know that $V(t) \geq \lambda_{\text {min }}\left(P_{1}\right)\|x(t)\|^{2}$. Let $M=\left(V\left(t_{0}\right) / \lambda_{\text {min }}\left(P_{1}\right)\right)^{\frac{1}{2}}$, then

$$
\|x(t)\| \leq M e^{-\frac{1}{2} \alpha\left(t-t_{0}\right)}, \quad t \geq t_{0}
$$

Inequality (21) implies model (3) is globally exponentially stable. The proof is complete. 


\section{Example}

In this section, we will provide an example to illustrate the effectiveness of the obtained result. Consider a 2-dimensional neural networks (1), where

$$
\begin{gathered}
D=\left(\begin{array}{cc}
0.8 & 0 \\
0 & 0.9
\end{array}\right), \quad A=\left(\begin{array}{cc}
0.1+0.3 i & -0.4-0.6 i \\
-0.2-0.6 i & -1+i
\end{array}\right), B=\left(\begin{array}{cc}
0.7+0.2 i & 0.4-i \\
-0.1+0 . i & 0.5-i
\end{array}\right), \\
f_{1}(x)=f_{2}(x)=\frac{1}{20}(|x-1|-|x+1|), \tau(t)=8|\sin (t)| .
\end{gathered}
$$

It is easy to see that $\tau(t)=8$, and Assumption 1 is satisfied with $L=\left(\begin{array}{cc}0.1 & 0 \\ 0 & 0.1\end{array}\right)$. Taking $\alpha=0.1$, by the YALMIP toolbox in MATLAB , we can find a solution to the complex-valued linear matrix inequality (5) as follows:

$$
\begin{aligned}
& P_{1}=\left(\begin{array}{cc}
77.1925 & -3.9825+0.1936 i \\
-3.9825-0.1936 i & 68.9255
\end{array}\right), \quad P_{2}=\left(\begin{array}{cc}
57.4822 & 0.5662-0.1293 i \\
0.5662+0.1293 i & 57.9773
\end{array}\right), \\
& P_{3}=\left(\begin{array}{cc}
1.8901 & -0.4011+0.0295 i \\
-0.4011-0.0295 i & 1.0653
\end{array}\right), \quad R=\left(\begin{array}{cc}
77.4715 & 0 \\
0 & 112.5789
\end{array}\right) \text {, } \\
& S=\left(\begin{array}{cc}
84.0433 & 0 \\
0 & 81.2348
\end{array}\right), \quad Q_{1}=\left(\begin{array}{cc}
0.2872-0.0013 i & -0.0712+0.0058 i \\
-0.0827-0.0089 i & 0.1430+0.0013 i
\end{array}\right), \\
& Q_{2}=\left(\begin{array}{cc}
1.8237-0.0008 i & -0.3858+0.0237 i \\
-0.3896-0.0249 i & 1.0076+0.0005 i
\end{array}\right), W=\left(\begin{array}{cc}
31.1013-0.0454 i & -6.3074+0.2873 i \\
-6.2808-0.2961 i & 17.8358+0.0307 i
\end{array}\right), \\
& Z=\left(\begin{array}{cc}
-43.6642+0.0171 i & -4.1023+0.2865 i \\
-3.4447-0.2847 i & -48.6779-0.0075 i
\end{array}\right) \text {. }
\end{aligned}
$$

Therefore, by Theorem 1, we know that the origin of system (3) is globally exponerntially stable, and the gain matrix of control law (2) is

$$
K=W^{-1} Z=\left(\begin{array}{ll}
-1.5545-0.0033 i & -0.7382+0.0369 i \\
-0.7406-0.0417 i & -2.9898+0.0054 i
\end{array}\right) \text {. }
$$

The numerical simulation is shown in figure 1 and figure 2.
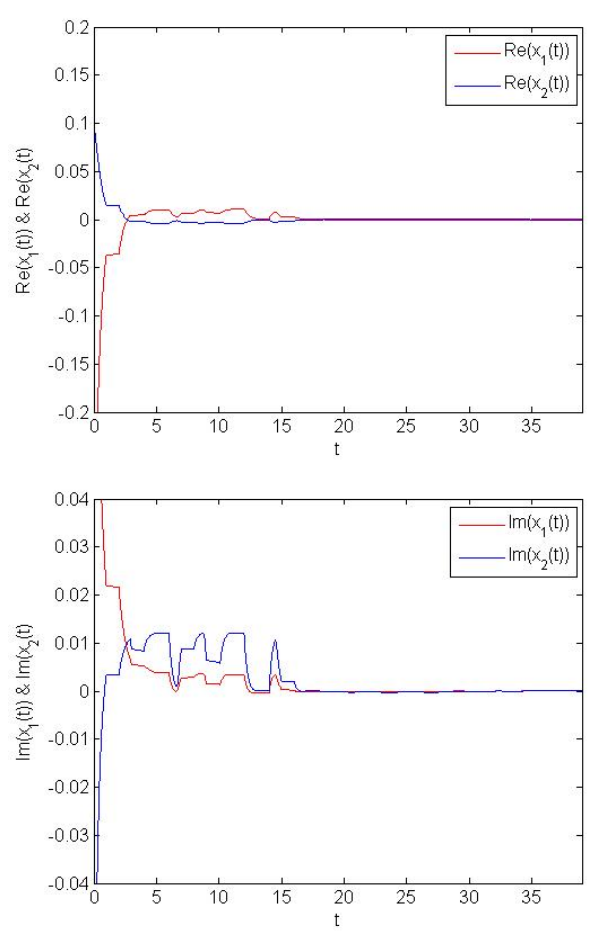


\section{Conclusions}

In this paper, the stabilization problem for a class of complex-valued neural networks with time-varying delay has been investigated. A delay-dependent criterion for checking the stability of the considered neural networks has been obtained by constructing proper Lyapunov-Krasovskii functional and using the matrix inequality techniques. An example with simulations is also given to show the effectiveness of the obtained result.

\section{Acknowledgments}

This work was supported by the National Natural Science Foundation of China under Grants 61273021, 61473332 and 11402214, and in part by the Natural Science Foundation Project of CQ cstc2013jjB40008.

\section{References}

[1] O.M. Kwon, M.J Park, S.M. Lee, J.H. Park, and E.J. Cha, Stability for neural networks with time-varying delays via some new approaches. IEEE Transactions on Neural Networks and Learning Systems 24(2013), p. 181-193.

[2] J.D. Cao and Q.K. Song, Stability in Cohen-Grossberg-type bidirectional associative memory neural networks with time-varying delays. Nonlinearity 19(2006), p. 1601-1617.

[3] Q.K. Song and J.D. Cao, Impulsive effects on stability of fuzzy Cohen-Grossberg neural networks with time-varying delays. IEEE Transactions on Systems, Man, and Cybernetics 37(2007), p. 733-741.

[4] Q.K. Song and J.Y. Zhang, Global exponential stability of impulsive Cohen-Grossberg neural network with time-varying delays. Nonlinear Analysis: Real World Applications 9(2008), p. 500-510.

[5] Q.K. Song and J.Y. Zhang, Global exponential stability of impulsive Cohen-Grossberg neural network with time-varying delays. Nonlinear Analysis: Real World Applications 9(2008), p. 500-510.

[6] H.G.Zhang, Z.S.Wang and D.R. Liu, A comprehensive review of stability analysis of continuous-time recurrent neural networks. IEEE Transactions on Neural Networks and Learning Systems 25(2014), p. 1229-1262.

[7] A. Hirose, Dynamics of fully complex-valued neural networks. Electronics Letter, 28(1992) p. 1492-1494.

[8] D. Lee, Relaxation of the stability condition of the complex-valued neural networks. IEEE Transactions on Neural Networks 12(2001), p. 1260-1262.

[9] T. Nishikawa, T. Iritani, K. Sakakibara and Y. Kuroe, Phase dynamics of complex-valued neural networks and its application to traffic signal control. International Journal of Neural Systems 15(2005), p. 111-120.

[10] D. Mishra, A. Tolambiya, A. Shukla, and P. Kalra, Stability analysis for higher order complex-valued Hopfield neural network. Lecture Notes in Computer Science 4232(2006), p. 608-615.

[11] V. Rao and G. Murthy, Global dynamics of a class of complex valued neural networks. International Journal of Neural Systems 18(2008), p. 165-171. 
[12] C.J. Duan and Q.K. Song, Boundedness and stability for discrete-time delayed neural network with complex-valued linear threshold neurons. Discrete Dynamics in Nature and Society 2010(2010), Article ID 368379.

[13] J. Hu and J. Wang, Global stability of complex-valued recurrent neural networks with time-delays. IEEE Transactions on Neural Networks and Learning Systems 23(2012), p. 853-865.

[14] B. Zhou and Q.K. Song, Boundedness and complete stability of complex-valued neural networks with time delay. IEEE Transactions on Neural Networks and Learning Systems 24(2013), p. 1227-1238.

[15] X.F. Chen and Q.K. Song, Global stability of complex-valued neural networks with both leakage time delay and discrete time delay on time scales. Neurocomputing 121(2013), p. 254-264.

[16] Z.Y. Zhang, C. Lin and B. Chen, Global stability criterion for delayed complex-valued recurrent neural networks. IEEE Transactions on Neural Networks and Learning Systems 25(2014), p. 1704-1708.

[17] T. Fang and J.T. Sun, Stability of complex-valued recurrent neural networks with time-delays. IEEE Transactions on Neural Networks and Learning Systems 25(2014), p. 1709-1713.

[18] X.H. Xu, J.Y.Zhang, and J.Z. Shi, Exponential stability of complex-valued neural networks with mixed delays. Neurocomputing 128(2014), p. 483-490.

[19] X.F. Chen, Q.K. Song, Y.R. Liu, and Z.J.Zhang, Global $\mu$-stability of impulsive complex-valued neural networks with leakage delay and mixed delays. Abstract and Applied Analysis 2014(2014), Article ID 397532.

[20] X.F. Chen, Q.K. Song, X.H. Liu, and Z.J.Zhang, Global $\mu$-stability of complex-valued neural networks with unbounded time-varying delays. Abstract and Applied Analysis 2014(2014), Article ID ID 263847.

[21] Q.K. Song, Z.J.Zhang, and Y.R. Liu, Stability analysis of complex-valued neural networks with probabilistic time-varying delays. Neurocomputing 159(2015), p. 96-104.

[22] G. Velmurugan, R. Rakkiyappan, and J.D. Cao, Further analysis of global $\mu$-stability of complex-valued neural networks with unbounded time-varying delays. Neural Networks 67(2015), p. 14-27. 